PROCEEDINGS OF THE

AMERICAN MATHEMATICAL SOCIETY

Volume 131, Number 12, Pages 3647-3656

S 0002-9939(03)06937-5

Article electronically published on February 26, 2003

\title{
A PROOF OF THE GENERALIZED BANACH CONTRACTION CONJECTURE
}

\author{
ALEXANDER D. ARVANITAKIS
}

(Communicated by John R. Stembridge)

\begin{abstract}
We introduce the notion of $J$-continuity, which generalizes both continuity and the hypothesis in the Generalized Banach Contraction Conjecture, and prove that any $J$-continuous self-map on a scattered compact space, has an invariant finite set. We use the results and the techniques to prove the Generalized Banach Contraction Conjecture.
\end{abstract}

\section{INTRODUCTION}

The Banach Contraction Principle is one of the most important and heavily investigated fixed point theorems. The following conjecture generalizes the one originally studied by Banach.

Generalized Banach Contraction Conjecture (GBCC). Let $T: X \rightarrow X$ be a self-map of a complete metric space $(X, d)$, and let $0<M<1$. Let $J$ be a positive integer. Assume that for each pair $x, y \in X$,

$$
\min \left\{d\left(T^{k} x, T^{k} y\right): 1 \leq k \leq J\right\} \leq M d(x, y) .
$$

Then $T$ has a fixed point.

Banach's original theorem is simply the case $J=1$, in which $T$ is uniformly continuous.

Below, we briefly summarize what has been proved about GBCC until now.

In 2], it is proved that GBCC is true if $J=2$ without any additional assumption on $T$. Moreover it is true for $J=3$ with the additional assumption that $T$ is continuous. It is also shown that the case $J=3$ includes examples where $T$ may be discontinuous.

In [3], it is proved that GBCC is true for arbitrary $J$, if one assumes that $T$ is uniformly continuous.

In [6], it is shown that GBCC is true for arbitrary $J$, with the additional assumption that $T$ is strongly continuous.

Finally, in [4], it is shown that GBCC is true if $T$ is continuous. Moreover it is proved for $J=3$, without any additional assumption on $T$.

Received by the editors May 22, 2002 and, in revised form, July 9, 2002.

2000 Mathematics Subject Classification. Primary 05C55, 47H10.

Key words and phrases. Fixed point, GBCC, Banach Contraction Principle, scattered compact. 
In this paper, we establish the GBCC. The proof may be divided into two parts. The results of both parts are conclusions of statements for relations on the integers (Lemma 3.2 and Lemma 4.2). Thus the proof is mostly of a combinatorial nature.

In the first part, which is proved in section 4, we strengthen [4 Proposition 1]. Namely, we prove that if $\left\{x_{n}\right\}_{n}$ is a sequence in a metric space, and there are $J \in \mathbb{N}$ and $0<M<1$ such that

$$
\min \left\{d\left(x_{n+k}, x_{m+k}\right): 1 \leq k \leq J\right\} \leq M d\left(x_{n}, x_{m}\right) \text { for all integers } n \text { and } m,
$$

then there is a piecewise syndetic sequence $n_{1}<n_{2}<\ldots$ such that $\left\{x_{n_{i}}\right\}_{i}$ is Cauchy.

Here, a subset of $\mathbb{N}$ is said to be piecewise syndetic if there exists an $N \in \mathbb{N}$ and arbitrarily large finite subsets of it, say $n_{1}<n_{2}<\ldots<n_{k}$ such that $n_{i+1}-n_{i} \leq N$ for all $i$ such that $1 \leq i \leq k-1$. (See also [1. Definition 1.11] or Definition 4.1.)

In the second part, which is proved in sections 2 and 3 , we prove that if $\left\{n_{i}\right\}_{i}$ is a piecewise syndetic sequence, $(X, d), T$ satisfy the GBCC assumption, and there are $z, \omega \in X$ such that $T^{n_{i}} z \rightarrow \omega$, then there is a finite subset of $\left\{T^{k} \omega: k \in \mathbb{N}\right\}$ which is $T$-invariant. This, in conjunction with [2, Lemma 1], results in a fixed point for $T$. We essentially prove something more general than this which has some interesting consequences on scattered compact spaces. In order to describe these consequences, we first need some notation.

Let $(X, d)$ be a metric space and $T: X \rightarrow X$ a self-map. Throughout this paper, we will say that $T$ satisfies the $G B C C$ assumption, if for each pair $x, y \in X$,

$$
\min \left\{d\left(T^{k} x, T^{k} y\right): 1 \leq k \leq J\right\} \leq M d(x, y) .
$$

So, GBCC states that if $T$ satisfies the GBCC assumption and $X$ is complete, then $T$ has a fixed point.

Definition 1.1. Let $X$ be a topological space, $J \in \mathbb{N}$ and $T: X \rightarrow X$ a self-map. We say that $T$ is $J$-continuous if for every $x \in X$ and every choice of neighborhoods $U_{i} \ni T^{i} x, 1 \leq i \leq J$, there is a neighborhood $U \ni x$ such that if $y \in U$, then there are $1 \leq i, j \leq J$ so that $T^{j} y \in U_{i}$.

Clearly the case where $T$ is continuous is the case of 1 -continuous according to the above definition. Moreover the notion of $J$-continuity generalizes the GBCC assumption: If $T$ satisfies the GBCC assumption and $U_{i}=B\left(T^{i} x, \varepsilon_{i}\right)$ (i.e. $U_{i}$ is the ball with center $T^{i} x$ and radius $\left.\varepsilon_{i}\right)$, then we can simply take $U=B\left(x, \frac{\min \left\{\varepsilon_{i}: 1 \leq i \leq J\right\}}{M+1}\right)$.

Observe that the notion of $J$-continuity generalizes GBCC assumption in two aspects: The first is analogous to the generalization of the contraction by continuity. The second is that we do not require $T^{i} x$ and $T^{j} y$ to be "close enough" for the same iterate of $T$.

Clearly since continuous maps are $J$-continuous, it is not necessary for a $J$ continuous self-map to have a fixed point. But in any case we prove the following:

Theorem 1.1. If $X$ is a scattered compact space (not necessarily metric) and $T: X \rightarrow X$ is $J$-continuous, then there exists a finite $A \subset X$ which is $T$-invariant, i.e. $T[A] \subset A$.

We recall that a compact space $X$ is called scattered if every non-empty closed subset $M$ of $X$ has a relatively isolated point, i.e. there exists an $x \in M$ and an open set $U \subset X$ such that $U \cap M=\{x\}$. 
Theorem [1.1] is the best possible in this direction, since it is easy to construct a scattered compact space and a continuous self-map of it having no fixed points. The simpler case is to take $X=\left\{x_{1}, \ldots, x_{n}\right\}$, a finite set with the discrete topology, and

$$
T\left(x_{i}\right)= \begin{cases}x_{i+1} & \text { if } 1 \leq i<n, \\ x_{1} & \text { if } i=n .\end{cases}
$$

According to [2, Lemma 1], if $T$ satisfies the GBCC assumption and $A$ is finite and $T$-invariant, then $T$ has a fixed point. So in particular Theorem 1.1 easily implies the GBCC in the case where $X$ is a scattered compact metric space.

Since the case where $T$ is continuous is from many aspects analogous to but simpler than the general one, we present it first in section 2 .

In section 3, we give the general proof of Theorem 1.1.

Finally in section 4 we give the proof of the full GBCC.

I would like to thank Professor S. A. Argyros for bringing to my attention the original problem and for comments while preparing the paper. I also thank Professor Ch. Verykios for suggestions that improved and clarified the proof of Lemma 4.1.

While finishing the editing of the paper, I was informed by Professor J. Stein that he and Professor J. Merryfield have also obtained a proof for GBCC in [5].

\section{The CONTINUOUS CASE FOR SCATTERED COMPACT}

Let us briefly recall some facts from the theory of scattered compact spaces. Let $X$ be a scattered compact. For $\alpha$ ranging in the ordinals, we define the derivative sets $X^{(\alpha)}$ by recursion on $\alpha$. Then

- $X^{(0)}=X$.

- If $\alpha=\beta+1, X^{(\beta+1)}=X^{(\beta)} \backslash\left\{x \in X^{(\beta)}: x\right.$ is an isolated point of $\left.X^{(\beta)}\right\}$ and

- if $\alpha$ is a limit ordinal $X^{(\alpha)}=\bigcap_{\beta<\alpha} X^{(\beta)}$.

For any closed subset $M$ of $X$, we denote by $M^{(\alpha)}$ the set $M \cap X^{(\alpha)}$. Since $X^{(\alpha)}$ is always a closed subset of $X$, if $X^{(\alpha)} \neq \emptyset$, then $X^{(\alpha+1)} \subsetneq X^{(\alpha)}$ by the definition of scattered, so that there always exists an ordinal $\alpha$ such that $M^{(\alpha)}=\emptyset$. If $\alpha_{0}$ is the least such $\alpha$, then by the compactness of $X$ and $M$ this must be a successor ordinal. We denote by $\alpha(M)$ the ordinal $\alpha_{0}-1$. Again using a compactness argument, we see that $M^{\left(\alpha_{0}-1\right)}$ must be a finite set. For an element $x$ of $X, \alpha(\{x\})$ will also be denoted by $\alpha(x)$ for simplicity.

We begin with the following lemma:

Lemma 2.1. Let $X$ be a $T_{2}$ space and $T: X \rightarrow X$ a continuous map having the property that every finite subset $A$ of $X$ is not $T$ invariant, namely $T[A] \not \subset A$. Then for every $N \in \mathbb{N}$ and for every $z \in x$ there exists a neighborhood $U_{z}$ of $z$ such that for every $2 N$ successive iterates of $T$, starting at any point $y, y, T y, \ldots, T^{2 N-1} y, N$ successive ones among them do not belong to $U_{z}$. That is, there exists a $0 \leq n_{0} \leq$ $N-1$, such that for all $i$ such that $1 \leq i \leq N, T^{n_{0}+i} y \notin U_{z}$.

Proof. For $N \in \mathbb{N}$ and $z \in X$, consider the following points: $z, T z, T^{2} z, \ldots, T^{N} z$. These are $N+1$ distinct points of $X$ by our hypothesis that there exists no finite $T$-invariant subset of $X$. Using induction on $i$, we define an open neighborhood $U_{N-i}$ of $T^{N-i} z$ as follows: $U_{N}$ is any open neighborhood of $T^{N} z$ such that 
$z, T z, \ldots, T^{N-1} z \notin \bar{U}_{N}$. (Here, by $\bar{U}_{N}$ we denote the closure of $U_{N}$.) Having defined $U_{N}, \ldots, U_{N-i}$, for $i<N$, we define $U_{N-(i+1)}$ such that

(1) $U_{N-(i+1)} \cap U_{j}=\emptyset$ for $N-i \leq j \leq N$.

(2) $z, T z, \ldots, T^{N-(i+2)} z \notin \bar{U}_{N-(i+1)}$.

(3) For every $x \in U_{N-(i+1)}, T x \in U_{N-i}$.

We can find such a neighborhood of $T^{N-(i+1)} z$, since $X$ is a $T_{2}$ space, $T$ is continuous and $T\left(T^{N-(i+1)} z\right)=T^{N-i} z \in U_{N-i}$.

The crucial properties of $U_{0}, \ldots, U_{N}$ are that they are pairwise disjoint, and moreover $T\left[U_{i}\right] \subset U_{i+1}$. So, if for some $x, x \in U_{0}$, then for all $i$ in the range $1 \leq i \leq N, T^{i} x \in U_{i}$ and hence $T^{i} x \notin U_{0}$.

Now set $U_{z}=U_{0}$ and given $y \in X$, consider $2 N$ successive iterates of $T$ on $y$, $y, T y, \ldots, T^{2 N-1} y$. If all of $y, T y, \ldots, T^{N-1} y$, do not belong to $U_{z}$, then we have nothing to prove. Otherwise there exists a $0 \leq n_{0} \leq N-1$ such that $T^{n_{0}} y \in U_{z}$. But in this case all of $T^{n_{0}+i} y, 1 \leq i \leq N$, do not belong to $U_{z}=U_{0}$, since $T^{n_{0}+i} y \in U_{i}$ and $U_{i} \cap U_{0}=\emptyset$.

Remark 2.1. It is easy to see that in order to find for a particular $z$ the required neighborhood $U_{z}$, it suffices to know that $z, T z, T^{2} z, \ldots, T^{N} z$ are all distinct. So, if for some fixed $z$ and $N$ there is no such neighborhood, then there exists an $A \subset\left\{z, T z, \ldots, T^{N} z\right\}$ which is $T$-invariant. In particular, the same proof is possible if we weaken the hypothesis to the following: There is no $T$-invariant set $A$ of cardinality $|A| \leq N$.

The following theorem is a case of Theorem 1.1 in which $T$ is 1-continuous.

Theorem 2.2. Let $X$ be a scattered compact space and $T: X \rightarrow X$ a continuous map. Then there exists a finite $A \subset X$ which is $T$-invariant, that is, $T[A] \subset A$.

Proof. We will prove it by contradiction. So assume there is not any. In particular $T$ and $X$ satisfy the hypothesis of Lemma 2.1. For any $N \in \mathbb{N}$ denote by $p(N)=2 N$ the sufficient number of successive iterates of $T$ that contain $N$ successive ones among them not belonging to $U_{z}$ (using the notation of Lemma 2.1).

We define inductively $z_{1}, z_{2}, \ldots$ points of $X, U_{1}, U_{2}, \ldots$ neighborhoods of these points, and $\alpha_{1}=\alpha\left(z_{1}\right), \alpha_{2}=\alpha\left(z_{2}\right), \ldots$ ordinal numbers as follows: Having defined them until $m$ ( $m$ may be 0 ), we consider the closed subspace of $X, X_{m+1}=X \backslash$ $\bigcup_{i=1}^{m} U_{i}$ and let $\alpha_{m+1}=\alpha\left(X_{m+1}\right)$. Next, if $X_{m+1} \neq \emptyset$, we find a $z_{m+1} \in X_{m+1}$ with $\alpha\left(z_{m+1}\right)=\alpha_{m+1}$ and $U_{m+1}$ will be the neighborhood of $z_{m+1}$, according to Lemma 2.1] that works for $N=p^{m}(1)$. We prove the following:

Claim. The sequence $\left\{\alpha_{m}\right\}_{m}$ is a non-increasing sequence of ordinals, and moreover if $X_{m} \neq \emptyset$ for all $m \in \mathbb{N}$, then for all $m \in \mathbb{N}$, there exists an $m^{\prime}>m$ such that $\alpha_{m^{\prime}}<\alpha_{m}$.

The first is obvious, since $X_{m+1} \subset X_{m}$. Moreover, fixing $m \in \mathbb{N}$, there are just finitely many points $x$ of $X_{m}$ such that $\alpha(x)=\alpha\left(X_{m}\right)$. Therefore if for all $m^{\prime}>m, \alpha_{m^{\prime}}=\alpha_{m}$, then for all $m^{\prime}>m, \alpha\left(z_{m^{\prime}}\right)=\alpha_{m}$ and $z_{m^{\prime}} \in X_{m}$. This is a contradiction, since it follows easily from their construction that $\left\{z_{m}\right\}_{m}$ are all distinct. 
Since there is no strictly decreasing sequence of ordinals, the claim easily implies that there exists an $m \in \mathbb{N}$ such that $X_{m+1}=\emptyset$. Therefore

$$
X=\bigcup_{i=1}^{m} U_{i}
$$

Fix an $x \in X$ and consider the following first $p^{m}(1)+1$ successive iterates of $T$ on $x: x, T x, \ldots, T^{p^{m}(1)} x$. By the definition of $p^{m}(1)=p\left(p^{m-1}(1)\right)$ and of the neighborhood $U_{m} \ni z_{m}$, there are $p^{m-1}(1)$ successive ones among them not belonging to $U_{m}$, so there are $p^{m-2}(1)$ successive ones among them not belonging to $U_{m-1} \cup U_{m}$. Continuing this way, we find that there exists an element among $x, T x, \ldots, T^{p^{m}(1)-1} x$ not belonging to $\bigcup_{i=1}^{m} U_{i}$ which is a contradiction by (11).

\section{The general $J$-COntinuous CASE}

It is easy to check that the same proof could be used to prove Theorem 1.1 provided that we had proved an analog to Lemma 2.1 for the general $J$-continuous case. So, in order to prove Theorem 1.1 we only need the following lemma:

Lemma 3.1. Let $X$ be a $T_{2}$-space and $T: X \rightarrow X$ a J-continuous map having the property that every finite $A$ subset of $X$ is not $T$-invariant. Then for every $N \in \mathbb{N}$, there exists a $p(N) \in \mathbb{N}$ such that every $z \in X$ has a neighborhood $U_{z}$ with the following property: If $y, T y, \ldots, T^{p(N)-1} y$ are any $p(N)$ successive iterates of $T$, then there are $N$ successive ones among them not belonging to $U_{z}$.

We will try to imitate the proof of Lemma 2.1. We will need the following technical lemma, in which the relation $R$ must be thought of as

$$
i R j \Longleftrightarrow T^{i} x \in U_{j} \text {, }
$$

using the notation of the proof of Lemma 2.1 This lemma is necessary in this particular case in order to calculate the appropriate number $p(N)$. We will use it to construct the neighborhoods $U_{i} \ni T^{i} z$ analogously to Lemma [2.1. We assume without loss of generality that $N=\ell J$ for some $\ell \in \mathbb{N}$.

Given $k, \ell \in \mathbb{N}$ we denote by $[k, \ell]$ the set of all $n \in \mathbb{N}$ such that $k \leq n \leq \ell$.

Lemma 3.2. Let $J, N=\ell J$, be given. Then for all $n, m \in \mathbb{N}$, there exists a number $p(n, m) \in \mathbb{N}$ such that whenever $R \subset[1, p(n, m)] \times[0, \infty)$ is a relation with the following properties

(1) in every interval $[k+1, k+N] \subset[1, p(n, m)]$ there exists an $i$ such that $i R 0$

(2) if $i+J \leq p(n, m)$ and $i R j$, then there are $1 \leq i^{\prime}, j^{\prime} \leq J$ such that $i+$ $i^{\prime} R j+j^{\prime}$,

then there exists a subinterval $[k+1, k+N] \subset[1, p(n, m)]$ and $k_{1}, \ldots, k_{n} \in \mathbb{N}$ such that

(1) $k_{r+1}-k_{r} \geq m, 1 \leq r \leq n-1$,

(2) for all $r$ in the range $1 \leq r \leq n$ there exists a $q \in[k+1, k+N]$ such that $q R k_{r}$.

Proof. We prove by induction on $n$ that there exists such a $p(n, m)$ for all $m$.

Easily, hypothesis (1) of the lemma implies that $N$ can serve as $p(1, m)$. Next, given the numbers $p(n, m), m \in \mathbb{N}$, we use our inductive hypothesis for $p\left(n, m^{\prime}\right)$, 
where $m^{\prime}=m+(m J+2 N) J$. We set

$$
p(n+1, m)=p\left(n, m^{\prime}\right)+m J+N .
$$

In order to prove that this works, let $R \subset[1, p(n+1, m)] \times[0, \infty)$ be a relation that satisfies properties (1) and (2). By the inductive hypothesis for $R$ 「 $\left[1, p\left(n, m^{\prime}\right)\right]$, there is an interval $[k+1, k+N] \subset\left[1, p\left(n, m^{\prime}\right)\right]$ and $k_{1}, \ldots, k_{n} \in \mathbb{N}$ such that (1) and (2) hold with $m$ replaced by $m^{\prime}$. For every $r$ in the range $1 \leq r \leq n$, let $q_{r} \in[k+1, k+N]$ be such that $q_{r} R k_{r}$. For every such $r$, we inductively define sequences $q_{r}^{1}=q_{r}, k_{r}^{1}=k_{r}$ and $q_{r}^{s+1}=q_{r}^{s}+i^{\prime}, k_{r}^{s+1}=k_{r}^{s}+j^{\prime}$ where $1 \leq i^{\prime}, j^{\prime} \leq J$ are such that $q_{r}^{s+1} R k_{r}^{s+1}$ granted that $q_{r}^{s} R k_{r}^{s}$, by property (2).

For any such $r$, let $s_{r}$ be an appropriate number such that

$$
q_{r}^{s_{r}} \in[k+N+m J+1, k+N+m J+N] .
$$

Since $k+N \leq p\left(n, m^{\prime}\right)$, this interval is a subinterval of $[1, p(n+1, m)]$.

Also let $q_{0}^{\prime}$ be in the same interval, according to (1), such that $q_{0}^{\prime} R 0$ and $k_{0}^{\prime}=0$, $k_{r}^{\prime}=k_{r}^{s_{r}}$. Notice that for any $1 \leq r \leq n$,

$$
\begin{aligned}
& m \leq s_{r} \leq m J+2 N \quad \text { so that } \\
& m \leq k_{r}^{s_{r}}-k_{r} \leq(m J+2 N) J .
\end{aligned}
$$

In particular

$$
\begin{aligned}
k_{1}^{\prime}-k_{0}^{\prime} & =k_{1}^{s_{1}} \geq m \quad \text { and } \\
k_{r+1}^{\prime}-k_{r}^{\prime} & =k_{r+1}^{s_{r+1}}-k_{r}^{s_{r}} \geq k_{r+1}-k_{r}-(m J+2 N) J \\
& \geq m^{\prime}-(m J+2 N) J=m .
\end{aligned}
$$

So $k_{0}^{\prime}, k_{1}^{\prime}, \ldots, k_{n}^{\prime}$ are the required numbers.

Now that we have Lemma 3.2 we can easily complete the proof of Theorem 1.1 by proving Lemma 3.1

Proof of Lemma 3.1. We will prove the lemma by contradiction, so we need $p(N)=$ $p(N+1,1)$ iterates of $T$ on $y$ and therefore the following $J p(N)+1$ iterates of $T$ on $z z, T z, \ldots, T^{J p(N)} z$ which are all distinct. For every $i$ in the range $0 \leq i \leq J p(N)$, we define a neighborhood $U_{i} \ni T^{i} z$ starting from the end and continuing inductively as in Lemma 2.1. in such a way that $U_{i}$ has the following properties:

(1) For every $j$ in the range $i<j \leq J p(N), U_{i} \cap U_{j}=\emptyset$.

(2) For every $j$ in the range $0 \leq j<i, \bar{U}_{i} \not \supset T^{j} z$.

(3) If $i+J \leq J p(N)$, then we also require, using the $J$-continuity of $T$, that whenever $x \in U_{i}$ then for some $1 \leq i^{\prime}, j^{\prime} \leq J, T^{j^{\prime}} x \in U_{i+i^{\prime}}$.

We set $U_{z}=U_{0}$ and given any $y \in X$ we define $R \subset[1, p(N)] \times[0, \infty)$ as follows:

$$
i R j \Longleftrightarrow T^{i-1} y \in U_{j} \text { and } j \leq i J .
$$

Assuming that the conclusion of the lemma is false for these particular $y, p(N)$ and $U_{z}$, in every interval $[k+1, k+N] \subset[1, p(N)]$ there must be an $i$ such that $T^{i-1} y \in U_{0}$ and trivially in this case $i R 0$. So property (1) of Lemma 3.2 is fulfilled for this particular $R$. Next we verify property (2): Assume that $i+J \leq p(N)$ and $i R j$. Since $j \leq i J$ we also have that $j+J \leq p(N) J$. Thus since $T^{i-1} y \in U_{j}$, there 
are $1 \leq i^{\prime}, j^{\prime} \leq J$ such that $T^{i+i^{\prime}-1} y \in U_{j+j^{\prime}}$. Moreover

$$
j+j^{\prime} \leq i J+J=(i+1) J \leq\left(i+i^{\prime}\right) J
$$

and thus $i+i^{\prime} R j+j^{\prime}$ as required.

Applying the conclusion of Lemma 3.2, there is a subinterval $[k+1, k+N] \subset$ $[1, p(N)]$ and (since $p(N)=p(N+1,1)) k_{1}, \ldots, k_{N+1} \in \mathbb{N}$ all distinct and for all $r$ such that $1 \leq r \leq N+1$, there is a $q \in[k+1, k+N]$ such that $q R k_{r}$. In this case there must be a $q \in[k+1, k+N]$ and $r \neq r^{\prime}$ so that $q R k_{r}$ and $q R k_{r^{\prime}}$. This means in particular that $T^{q-1} y \in U_{k_{r}}$ and $T^{q-1} y \in U_{k_{r^{\prime}}}$ which is impossible since by the choice of the neighborhoods $U_{i}, U_{k_{r}} \cap U_{k_{r^{\prime}}}=\emptyset$.

Remark 3.1. It is easy to see that Lemma 3.1 is closely related to Theorem 2 in [4]. Indeed, if we have obtained a sequence $n_{1}<n_{2}<\ldots$ of integers such that $n_{i+1}-n_{i} \leq N$ and $T^{n_{i}} x \rightarrow z$, but the iterates of $T$ on $z$ are all distinct, then for this particular $N$ we can find a neighborhood $U_{z} \ni z$ such that for any $y$ there is a $k,[k, k+N-1] \subset[0, p(N)]$ and $T^{k} y, \ldots, T^{k+N-1} y \notin U_{z}$. Setting $n_{i} \in \mathbb{N}$ such that for all $i^{\prime} \geq i, T^{n^{\prime}} x \in U_{z}$, we obtain a contradiction for $y=T^{n_{i}} x$.

\section{The proof of the GBCC}

We begin with the following definition (see also Definition 1.11 of [1]):

Definition 4.1. A finite set $A=\left\{n_{1}<n_{2}<\ldots<n_{k}\right\} \subset \mathbb{N}$ is called $N$-syndetic for some $N \in \mathbb{N}$ if $n_{i+1}-n_{i} \leq N$, for $1 \leq i \leq k-1$.

If $A$ is infinite, then it is called syndetic (or $N$-syndetic) if there exists an $N \in \mathbb{N}$ such that for all intervals of the form $[\ell+1, \ell+N]=\{i \in \mathbb{N}: \ell+1 \leq i \leq \ell+N\}$ we have that $[\ell+1, \ell+N] \cap A \neq \emptyset$.

Finally, an infinite $A \subset \mathbb{N}$ is called piecewise syndetic (or $N$-piecewise syndetic) if there exists an $N \in \mathbb{N}$ and arbitrarily large finite subsets of $A$ which are $N$ syndetic. That is, for all $k \in \mathbb{N}$, there exists a $B \subset A$, finite such that $|B| \geq k$ and $B$ is $N$-syndetic.

Let us recall from [1, Theorem 1.24] that iff $B$ is piecewise syndetic and we have partitioned it, $B=\bigcup_{i=1}^{n} B_{i}$, then for some $i, B_{i}$ is also piecewise syndetic.

The following lemma, combined with Lemma [3.1, [4, Lemma 1] and [2, Lemma $1]$, is the key lemma for proving the GBCC.

Lemma 4.1. Assume $(X, d)$ is a metric space, $T: X \rightarrow X$ is a self-map that satisfies $G B C C$ assumption for some $J$ and $M$, and $x, z \in X$. Assume moreover that there is a $K$-syndetic sequence $0<n_{1}<n_{2}<\ldots$ for some $K$ and a $C>0$ such that $d\left(T^{n_{i}} x, z\right)<C$ for all $i$. Then there is a piecewise syndetic sequence $m_{1}<m_{2}<\ldots$ such that $\left\{T^{m_{i}} z: i \in \mathbb{N}\right\}$ is Cauchy.

Before proving Lemma 4.1 let us first indicate how we can use it to derive a proof of the GBCC.

Proof of the GBCC. By Lemma 1 in [4, for any $z \in X$, there is a $J$-syndetic sequence $0<n_{1}<n_{2}<\ldots$ such that $\left\{T^{n_{i}} z\right\}$ is bounded. Thus Lemma 4.1 for $x=z$ implies that there exists an $N$ piecewise syndetic sequence $m_{1}<m_{2}<\ldots$ for some $N$, such that $\left\{T^{m_{i}} z\right\}_{i}$ is Cauchy. Let $\omega \in X$ be such that $T^{m_{i}} z \rightarrow \omega$. Assuming that $T$ has no fixed point, by [2, Lemma 1], $T$ cannot have any invariant finite set, i.e. there is no finite $A \subset X$ such that $T[A] \subset A$. Now using Lemma 3.1 we conclude that there is a neighborhood $U_{\omega}$ of $\omega$ and an integer $p(N)$ such that 
for any successive $p(N)$ iterates of $T, N$ successive ones among them are outside $U_{\omega}$.

Now, fix $i_{0} \in \mathbb{N}$ such that $T^{m_{i}} z \in U_{\omega}$ for all $i \geq i_{0}$. Using the definition of an $N$-piecewise syndetic sequence, there is a subset $\left\{m_{j+1}<m_{j+2}<\ldots<m_{j+k}\right\}$ of $\left\{m_{1}<m_{2}<\ldots\right\}$ of size $k=i_{0}+p(N)$ which is $N$-syndetic. Since in this case obviously $j+i_{0}, j+i_{0}+1, \ldots, j+i_{0}+p(N) \geq i_{0}$ we get that

$$
T^{m_{j+i_{0}}} z, T^{m_{j+i_{0}+1}} z, \ldots, T^{m_{j+i_{0}+p(N)}} z \in U_{\omega}
$$

and since for all $\ell$ such that $j+i_{0} \leq \ell \leq j+i_{0}+p(N)-1, m_{\ell+1}-m_{\ell} \leq N$, we derive that among the successive iterates $T^{i} z, m_{j+i_{0}} \leq i \leq m_{j+i_{0}+p(N)}$, there are no $N$ successive ones among them outside $U_{\omega}$. This arrives at a contradiction, since

$$
m_{j+i_{0}+p(N)}-m_{j+i_{0}} \geq j+i_{0}+p(N)-\left(j+i_{0}\right)=p(N)
$$

and the proof is complete.

The technique for proving Lemma 4.1 is in some sense similar to the one followed while proving Lemma 3.1. As in that case we will first need a technical lemma for some relation $R \subset \mathbb{N} \times \mathbb{N}$ which in this case must be thought of as

$$
i R j \Longleftrightarrow T^{i} x \in B\left(T^{j} z, r_{j}\right)
$$

where $B\left(T^{j} z, r_{j}\right)$ is the ball of radius $r_{j}$ centered in $T^{j} z$. We will fix the numbers $r_{j}$ later, in such a way that the conclusion of the lemma will give us a Cauchy subsequence of $\left\{T^{m} z\right\}_{m}$. The idea here is to use the triangle inequality in order to conclude that if for some $j_{1}, j_{2}$ and $i i R j_{1}$ and at the same time $i R j_{2}$, then $d\left(T^{j_{1}} z, T^{j_{2}} z\right)<r_{j_{1}}+r_{j_{2}}$.

Since a $K$-syndetic sequence is $K^{\prime}$-syndetic for all $K^{\prime}>K$, we may assume that $K \geq J$ in Lemma 4.1

Lemma 4.2. Given $J$ and $K \geq J$, assume that $R \subset \mathbb{N} \times[0, \infty)$ is a relation with the following properties:

(1) there is a $K$-syndetic sequence $n_{1}<n_{2}<\ldots$ such that for all $i, n_{i} R 0$.

(2) If for some $i$ and $j$ we have that $i R j$, then there exists an $1 \leq j^{\prime} \leq J$ such that $i+j^{\prime} R j+j^{\prime}$.

Then there is a piecewise syndetic sequence $m_{1}<m_{2}<\ldots$, every two elements of which are related with the same number, i.e. for all $m_{t}, m_{s}$ there exists an $i$ such that $i R m_{t}$ and $i R m_{s}$.

Proof. For $\ell \in[0, \infty)$ and $1 \leq s \leq K$, we define a set $L_{\ell}^{s}$ as follows:

$$
L_{\ell}^{s}=\{j: \ell K+s R j\}
$$

and we also define

$$
L_{\ell}=\bigcup_{s=1}^{k} L_{\ell}^{s}
$$

The crucial property of $L_{\ell}$ is the following:

Claim (1). Every $L_{\ell}$ has a $2 K$-syndetic finite subset $L_{\ell}^{\prime}$ such that $\min L_{\ell}^{\prime}=0$ and $\max L_{\ell}^{\prime} \geq(\ell-1) K$.

For every $i$, we define a strictly increasing and $J$-syndetic sequence $\left\{j_{i}(n)\right\}_{n}$ in such a way that

$$
n_{i}+j_{i}(n) R j_{i}(n) \quad \text { for all } n \text {. }
$$


To start with, we define $j_{i}(0)=0$. By property (1) of $R$, (3) holds with this definition. Next, assuming that we have defined $j_{i}(n)$, by property $(2)$, there is an $1 \leq j^{\prime} \leq J$ such that $n_{i}+j_{i}(n)+j^{\prime} R j_{i}(n)+j^{\prime}$. So we define $j_{i}(n+1)=j_{i}(n)+j^{\prime}$, so that $1 \leq j_{i}(n+1)-j_{i}(n) \leq J$ and (3) holds for $j_{i}(n+1)$.

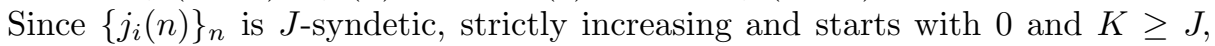
for every $\ell$ and $n_{i} \leq \ell K+K$, there exists an $n(\ell, i)$ such that $n_{i}+j_{i}(n(\ell, i)) \in$ $[\ell K+1, \ell K+K]$. In this case $j_{i}(n(\ell, i)) \in L_{\ell}$ by the definition of $L_{\ell}$ and (3). We will prove that $L_{\ell}^{\prime}=\left\{j_{i}(n(\ell, i)): n_{i} \leq \ell K+K\right\}$ has the required properties. Observe first that since $\left\{n_{i}\right\}_{i}$ is $J$-syndetic and $K \geq J$, there is an $i$ such that $n_{i} \in[\ell K+1, \ell K+K]$. For this particular $i, j_{i}(n(\ell, i))$ can be taken to be 0 and therefore $0 \in L_{\ell}^{\prime}$.

Next, since $n_{1}+j_{1}(n(\ell, 1)) \geq \ell K+1$ and $n_{1} \leq K$, we get that $j_{1}(n(\ell, 1)) \in L_{\ell}^{\prime}$ and $j_{1}(n(\ell, 1)) \geq(\ell-1) K$ as required.

In order to show that $L_{\ell}^{\prime}$ is $2 K$-syndetic, for $n_{i-1}, n_{i} \leq \ell K+K$, we wish to estimate the difference $j_{i-1}(n(\ell, i-1))-j_{i}(n(\ell, i))$. We first observe that since both terms $n_{i-1}+j_{i-1}(n(\ell, i-1))$ and $n_{i}+j_{i}(n(\ell, i))$ belong to the same interval $[\ell K+1, \ell K+K]$, we have that

$$
-K \leq n_{i-1}+j_{i-1}(n(\ell, i-1))-n_{i}-j_{i}(n(\ell, i)) \leq K,
$$

and moreover $1 \leq n_{i}-n_{i-1} \leq K$, since $\left\{n_{i}\right\}_{i}$ is $K$-syndetic. Therefore

$$
1-K \leq j_{i-1}(n(\ell, i-1))-j_{i}(n(\ell, i)) \leq 2 K .
$$

Now assume that $j_{i}(n(\ell, i))<j_{i^{\prime}}\left(n\left(\ell, i^{\prime}\right)\right)$ are two successive elements of $L_{\ell}^{\prime}$.

If $i<i^{\prime}$, then for the least $m>i$ such that $j_{i}(n(\ell, i))<j_{m}(n(\ell, m))$ we will have by (4) that $j_{m}(n(\ell, m))-j_{i}(n(\ell, i)) \leq K-1$. Therefore also $j_{i^{\prime}}\left(n\left(\ell, i^{\prime}\right)\right)-j_{i}(n(\ell, i)) \leq$ $K-1$.

If $i>i^{\prime}$, then for the largest $m<i$ such that $j_{i}(n(\ell, i))<j_{m}(n(\ell, m))$, again by (4) we will have that $j_{m}(n(\ell, m))-j_{i}(n(\ell, i)) \leq 2 K$ and therefore also $j_{i^{\prime}}\left(n\left(\ell, i^{\prime}\right)\right)-$ $j_{i}(n(\ell, i)) \leq 2 K$. In any case $L_{\ell}^{\prime}$ must be $2 K$-syndetic. This completes the proof of Claim (1).

Considering now every $L_{\ell}^{s}$ as an element of the compact space $\{0,1\}^{[0, \infty)}$ in the obvious way, we get that there exist a sequence $\ell_{1}<\ell_{2}<\ldots$ and $L_{\infty}^{s} \subset[0, \infty)$, $1 \leq s \leq K$, such that $\left\{L_{\ell_{m}}^{s}\right\}_{m} \rightarrow L_{\infty}^{s}$. Also set $L_{\infty}=\bigcup_{s=1}^{K} L_{\infty}^{s}$.

We will use Claim (1) to prove that

Claim (2). $L_{\infty}$ is a $2 K$-syndetic infinite sequence of $[0, \infty)$.

Let $[p+1, p+2 K]$ be any interval of size $2 K$. Using the convergence of $\left\{L_{\ell_{m}}^{s}\right\}_{m}$, we fix a big enough $m_{0}$ such that $\left(\ell_{m_{0}}-1\right) K \geq p+2 K$ and moreover for all $q \in[p+1, p+2 K]$ and $1 \leq s \leq K$,

$$
q \in L_{\ell_{m_{0}}}^{s} \Longleftrightarrow q \in L_{\infty}^{s} .
$$

By Claim (1), we get that $\max L_{\ell_{m_{0}}}^{\prime} \geq p+2 K$ and since $\min L_{\ell_{m_{0}}}^{\prime}=0$ and $L_{\ell_{m_{0}}}^{\prime}$ is $2 K$-syndetic, we get that $L_{\ell_{m_{0}}}^{\prime} \cap[p+1, p+2 K] \neq \emptyset$. Thus, since $L_{\ell_{m_{0}}}^{\prime} \subset L_{\ell_{m_{0}}}$, there must exist an $1 \leq s \leq K$ such that $L_{\ell_{m_{0}}}^{s} \cap[p+1, p+2 K] \neq \emptyset$. By (5- we also get that $L_{\infty}^{s} \cap[p+1, p+2 K] \neq \emptyset$ and hence $L_{\infty}$ is a $2 K$-syndetic infinite sequence as required. This completes the proof of Claim (2).

Using Theorem 1.24 of [1], as mentioned above, we get that there exists an $1 \leq s \leq K$ such that $L_{\infty}^{s}$ is piecewise syndetic. So, we only need to show that for every $j_{1}, j_{2} \in L_{\infty}^{s}$, there exists an $i \in \mathbb{N}$ such that $i R j_{1}$ and $i R j_{2}$. Fixing such 
elements $j_{1}, j_{2} \in L_{\infty}^{s}$, we find an $m_{0}$ such that $j_{1}, j_{2} \in L_{\ell_{m_{0}}}^{s}$. By the definition of $L_{\ell_{m_{0}}}^{s}$, (2), we get that $\ell_{m_{0}} K+s R j_{1}$ and $\ell m_{0} K+s R j_{2}$.

Using Lemma 4.2 we derive a proof of Lemma 4.1, thus concluding the proof of the GBCC.

Proof of Lemma 4.1]. Consider the relation $R \subset \mathbb{N} \times[0, \infty)$ given by the following rule:

$$
i R j \Longleftrightarrow T^{i} x \in B\left(T^{j} z, r_{j}\right), \quad \text { where } r_{j}=C \cdot M^{[j / J]}
$$

where $B\left(T^{j} z, r_{j}\right)$ is the $d$-ball centered at $T^{j} z$ with radius $r_{j}$ and by $[j / J]$ we denote the greatest integer below $j / J$. Since for all $i \in \mathbb{N}, d\left(T^{n_{i}} x, z\right)<C$, we get that for every $i, n_{i} R 0$. Therefore property (1) of Lemma 4.2 is fulfilled for the sequence $n_{1}<n_{2}<\ldots$ To prove property (2), assume that for some $i$ and $j, i R j$. Then $d\left(T^{i} x, T^{j} z\right)<r_{j}$ and since $T$ satisfies the GBCC assumption, there exists a $j^{\prime}$ in the range $1 \leq j^{\prime} \leq J$ such that $d\left(T^{i+j^{\prime}} x, T^{j+j^{\prime}} z\right)<r_{j} \cdot M$. Note that

$$
\begin{aligned}
r_{j} \cdot M & =C \cdot M^{[j / J]+1}=C \cdot M^{\left[\frac{j}{J}+1\right]} \\
& =C \cdot M^{\left[\frac{j+J}{J}\right]} \leq C \cdot M^{\left[\left(j+j^{\prime}\right) / J\right]} \\
& =r_{j+j^{\prime}} .
\end{aligned}
$$

Therefore $T^{i+j^{\prime}} x \in B\left(T^{j+j^{\prime}} z, r_{j+j^{\prime}}\right)$ in this case, so we get that $i+j^{\prime} R j+j^{\prime}$. Thus property (2) of Lemma 4.2 is also fulfilled. Using the conclusion of the lemma, we obtain a piecewise syndetic sequence $m_{1}<m_{2}<\ldots$ such that for every $t$ and $s$, there exists an $i$, so that both $i R m_{t}$ and $i R m_{s}$. In this case, by (6), $T^{i} x \in B\left(T^{m_{t}} z, r_{m_{t}}\right)$ and $T^{i} x \in B\left(T^{m_{s}} z, r_{m_{s}}\right)$. Using the triangle inequality, we get that

$$
d\left(T^{m_{t}} z, T^{m_{s}} z\right) \leq r_{m_{t}}+r_{m_{s}}=C\left(M^{\left[m_{t} / J\right]}+M^{\left[m_{s} / J\right]}\right)
$$

and the last inequality easily shows that indeed $\left\{T^{m_{i}} z\right\}_{i}$ is Cauchy.

\section{REFERENCES}

1. H. Furstenberg. Recurrence in Ergodic Theory and Combinatorial Number Theory. Princeton University Press, Princeton, 1981. MR 82j:28010

2. Jacek R. Jachymski, Bernd Schroder, and James D. Stein, Jr. A connection between fixed point theorems and tiling problems. J. Combin. Theory Ser. A, 87:273-286, 1999. MR 2000g:54075

3. Jacek R. Jachymski and James D. Stein, Jr. A minimum condition and some related fixed-point theorems. J. Austral. Math. Soc. Ser. A, 66:224-243, 1999. MR 2000a:54072

4. James Merryfield, Bruce Rothschild, and James D. Stein, Jr. An application of Ramsey's Theorem to the Banach contraction principle. Proceedings of the American Mathematical Society, 130(4):927-933, 2001. MR 2002h:54040

5. James Merryfield and James D. Stein, Jr. A generalization of the Banach Contraction Principle. to appear in Journal of Mathematical Analysis and Applications.

6. J. D. Stein, Jr. A systematic generalization procedure for fixed-point theorems. Rocky Mountain Journal of Mathematics., 30(2):735-754, 2000. MR 2001i:54052

MPla, Department of Mathematics, University of Athens, 15784 Panepistimiopolis, Athens, Greece

E-mail address: aarvan@cc.uoa.gr 